\title{
Imaging in Bone Metastases
}

\author{
(1) Nuri TASALI \\ Department of Radiology, Istanbul Oncology Hospital, Istanbul-Turkey
}

\section{Introduction}

Bone metastases are more prevalent than primary bone tumors. Apart from the liver and lungs, the most standard place of metastasis is the bones. Early diagnosis of bone metastases is critical in terms of correct staging and treatment planning in cancer patients. In the diagnosis of bone metastases and almost all radiological modalities, scintigraphy and hybrid imaging technique PET-CT (Positron Emission Tomography-Computed Tomography) is frequently used. With advancing technologies, the life expectancy of cancer patients has increased along with the increase in the incidence of bone metastases. According to recent post mortem studies, $70 \%$ of breast and prostate cancer patients have bone metastasis.[1]

Direct radiographs are mainly utilized to determine fracture risk in patients with existing bone metastases rather than diagnosing bone metastases. For metastases to be visible on direct radiographs, at least $50 \%$ of the affected bone tissue section must be damaged.[2] Lytic bone metastases are not well-circumscribed and do not have a thin sclerotic line around them, unlike benign lesions with lucent. Direct radiographs are not suitable for the diagnosis of bone metastases as well as their follow-up. It may take months for new bone formation in the bone tissue associated with recovery to become evident on direct radiography.

CT (Computed Tomography) is an extremely sensitive detection method, especially for sclerotic metastases. However, it may be slightly challenging to diagnose intramedullary lytic or slightly sclerotic lesions by CT (Fig. 1). Concerning all bone metastases, its sensitivity is at the level of $78 \%$, and its specificity is relatively low, with approximately 56\%.[3] It may be the best detection method for sclerotic metastases
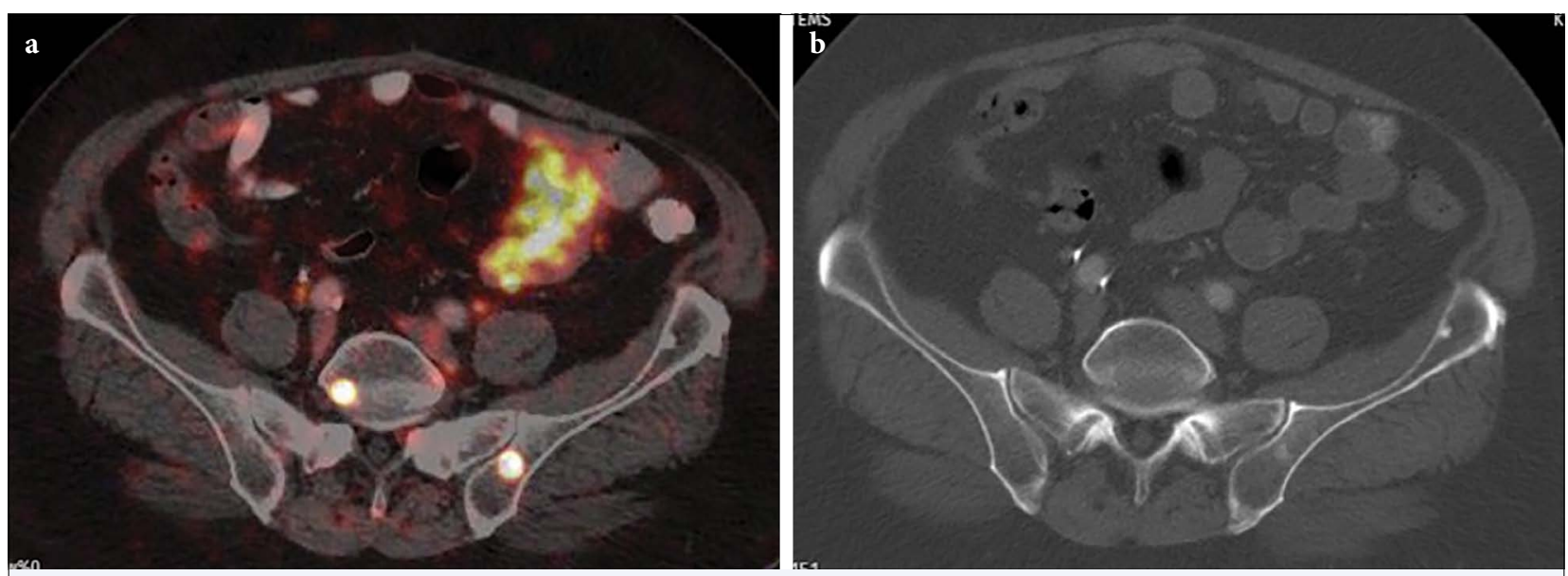

Fig. 1. (a) PET-CT fusion (b) CT bone window. The lesions having activity in the left iliac bone and the L5 vertebra in the PET-CT fusion can hardly be detected on the CT bone window.

Accessible online at: www.onkder.org

OPEN ACCESS This work is licensed under a Creative Commons Attribution-NonCommercial 4.0 International License.
Dr. Nuri TASALI

İstanbul Onkoloji Hastanesi,

Radyoloji Anabilim Dalı,

İstanbul-Turkey

E-mail: nuri.tasali@istanbulonko.com 
in thin bones such as ribs. It is also a known fact that fractures that develop due to metastases, especially in ribs, can be easily visualized with CT. Furthermore, it effectively monitors the healing of sclerosis in the treatment response (Fig. 2). It is crucial that bone metastases, which become visible with treatment, are not evaluated as new lesions.

MRI (Magnetic Resonance Imaging) is recognized as having the best soft-tissue resolution. It is also considered the most valuable method in the diagnosis of intramedullary lesions. Its sensitivity and specificity in detecting metastases is over $90 \%$. Another main advantage of MRI is that it does not use ionizing radiation.
In this context, it becomes the most reliable method for pregnant women and children. Another significant advantage of MRI is that it determines the relationship between bone metastases and neighboring soft tissues. Especially in vertebral metastases, determining the relationship between spinal cord and metastasis is extremely useful in pain management and radiotherapy treatment decisions. Revealing such anatomical details also contributes to radiotherapy treatment planning.

Lytic bone metastases display high signals in T2weighted sequences due to their high fluid content. Using fat suppression techniques in such cases to create a contrast difference in fat-containing medullary bone fa-
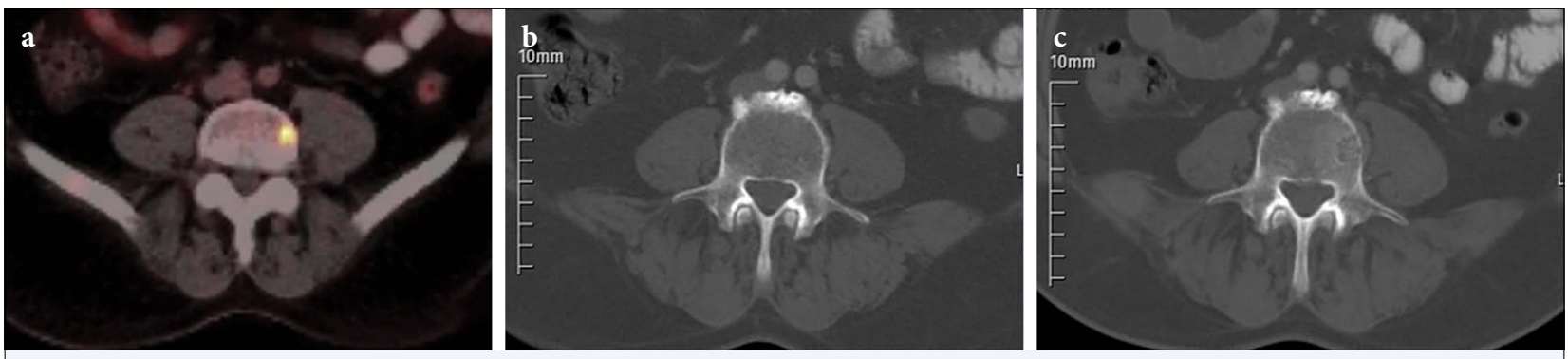

Fig. 2. (a) PET-CT fusion image June 2020 (b) CT bone window July 2020 (c) CT bone window February 2021 . The lesion seen in L4 vertebra in pre-treatment PET-CT cannot be distinguished in pre-treatment CT. In CT after chemotherapy, the lesion becomes selectable with new bone formation and sclerosis.
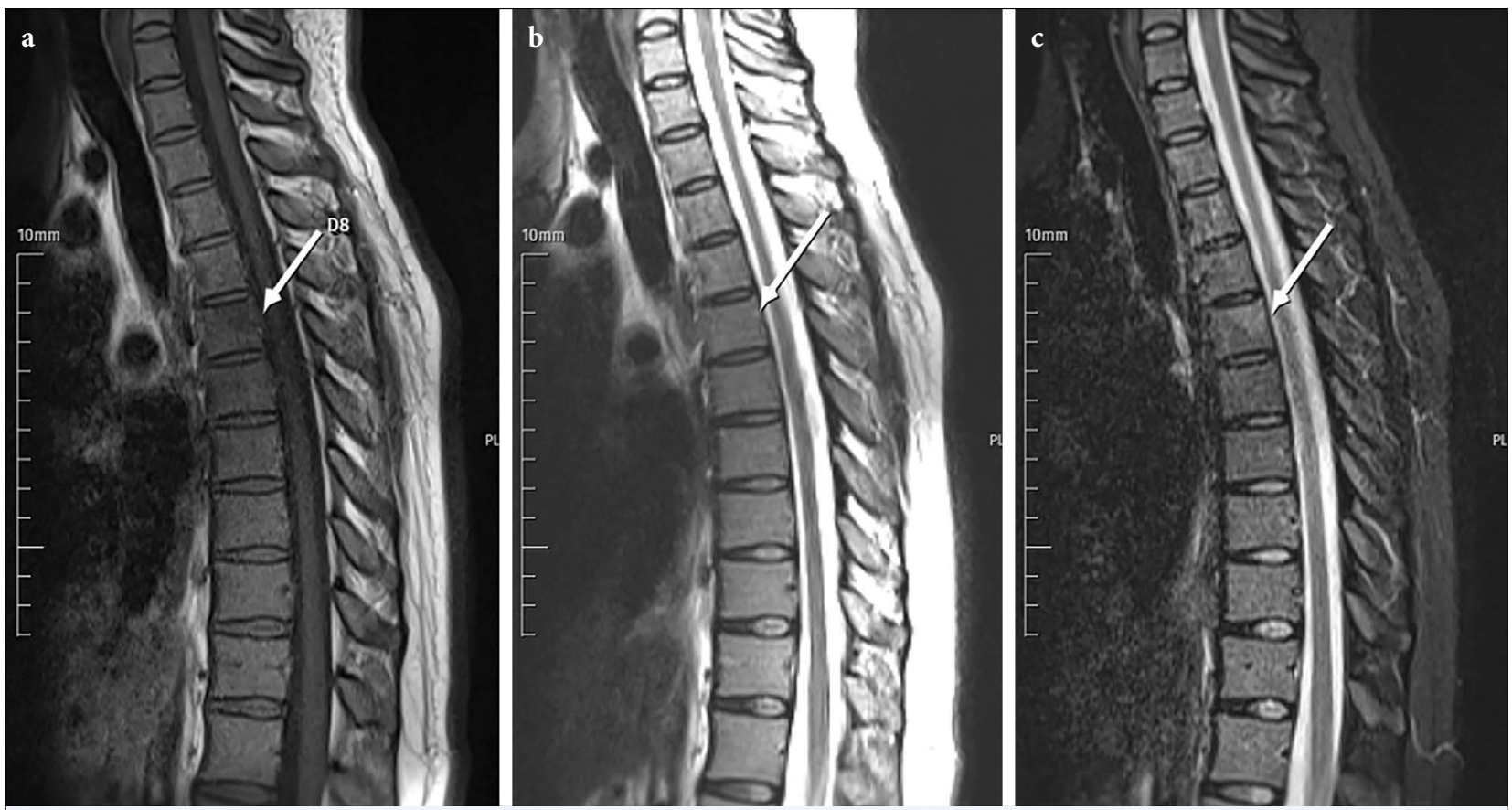

Fig. 3. (a) T1-weighted (b) T2-weighted (c) fat-suppressed STIR. The lytic metastasis observed in the T1-weighted sequence in the D8 vertebra cannot be differentiated in the T2-weighted sequence. The metastasis becomes visible with its fluid content on removing the fat signal from the medullary bone in the fat-suppressed STIR sequence. 
cilitates the identification of bone metastases. The STIR (Short Tau Inversion Recovery) sequence is a frequently used method in which fat is suppressed with a different technique to reveal bone metastases. Compared to T1weighted sequences, fat suppression techniques are used to create contrast with the medullary fatty bone (Fig. 3).

Since sclerotic metastases show low signal characteristics in both T1-and T2-weighted sequences, fat suppression techniques may not be required here. They can instead be easily diagnosed in non-contrast series with markedly low signal characteristics, especially in T1-weighted sequences. Contrast enhancement of sclerotic metastases also varies according to the degree of the sclerotic component. Therefore, non-contrast T1-weighted sequences are considered most valuable for diagnosis in sclerotic metastases.

It can be challenging to distinguish vertebral compression fractures as osteoporotic or metastatic. Although the best method for this is MRI, it is impossible to distinguish with total accuracy. It has been pointed out that in compression fractures, metastases should be considered if the bone marrow edema lasts more than three months. [4,5] Differentiation can be made in studies with diffusion-weighted MRI by looking at the changes in the extracellular fluid in compression fractures.

Diffusion-weighted imaging permits imaging of the whole body in a short time. This imaging technique, which generates signals according to the movement of the extracellular fluid, is often compared with bone scintigraphy and PET-CT. Since the loss of metastatic tumor cells with treatment increases diffusion in the extracellular area in the relevant region, this technique can be used to determine the treatment response.[6]

As the contribution of each imaging method to the questioned situation may be different, the diagnosis and follow-up of bone metastases require patient and cancer-specific imaging method choices. In conclusion, every viewing request starts with a question, and it should not be forgotten that this question can often be answered with findings from several imaging methods, especially for bone metastases.

\section{References}

1. Yu HH, Tsai YY, Hoffe SE. Overview of diagnosis and management of metastatic disease to bone. Cancer Control 2012;19(2):84-91.

2. Cuccurullo V, Cascini GL, Tamburrini O, Rotondo A, Mansi L. Bone metastases radiopharmaceuticals: an overview. Curr Radiopharm 2013;6(1):41-7.

3. O'Sullivan GJ, Carty FL, Cronin CG. Imaging of bone metastasis: An update. World J Radiol 2015;7(8):20211.

4. Shah LM, Salzman KL. Imaging of spinal metastatic disease. Int J Surg Oncol 2011;2011:769753.

5. Costelloe CM, Rohren EM, Madewell JE, Hamaoka T, Theriault RL, Yu TK, et al. Imaging bone metastases in breast cancer: techniques and recommendations for diagnosis. Lancet Oncol 2009;10(6):606-14.

6. Le Bihan D, Breton E, Lallemand D, Grenier P, Cabanis E, Laval-Jeantet M. MR imaging of intravoxel incoherent motions: application to diffusion and perfusion in neurologic disorders. Radiology 1986;161(2):401-7. 